\title{
Optimising the duration of antibiotic therapy for ventilator-associated pneumonia
}

\author{
J. Chastre and C.E. Luyt
}

ABSTRACT: Ventilator-associated pneumonia (VAP) has traditionally been treated with a 14-21day course of antibiotics. However, prolonged antibiotic therapy is associated with the emergence of multidrug-resistant strains, as well as higher toxicity and costs.

In a large, randomised, controlled trial in patients with microbiologically confirmed VAP who received appropriate empirical antibiotic therapy, an 8-day antibiotic regimen was not associated with excess mortality or more episodes of recurrent pulmonary infection compared with a 15-day regimen.

Amongst patients who developed recurrent infection, multidrug-resistant pathogens emerged less frequently in the group receiving 8 days of antibiotic therapy. The 8-day regimen was also not associated with excess mortality in the subgroup with VAP caused by nonfermentative Gram-negative bacilli, mostly Pseudomonas aeruginosa, although recurrent infections occurred more often.

Pending confirmatory studies, an 8-day course of antibiotic therapy may be appropriate for many patients with ventilator-associated pneumonia, providing that initial antibiotic therapy is appropriate, the clinical course is favourable and extreme vigilance is maintained after stopping antibiotics. Patients whose initial treatment regimen was inappropriate, those infected with difficult-to-treat pathogens such as Pseudomonas aeruginosa, and immunocompromised patients and others at high risk for relapse are likely to require a longer duration of antibiotic therapy.

KEYWORDS: Antibiotic therapy duration, intensive care unit, resistance, ventilator-associated pneumonia

$\mathbf{T}$ he emergence and rapid dissemination of multidrug-resistant bacterial pathogens are a continually growing threat in hospitals, especially in intensive care units (ICUs). Three pathogens are particularly problematic: Pseudomonas aeruginosa, Acinetobacter species and methicillinresistant Staphylococcus aureus (MRSA). Numerous surveillance programmes have tracked antibiotic susceptibility profiles in these organisms and shown that a large proportion of isolates are now resistant to once widely effective antibiotics. This point is illustrated by an analysis of $>50,000$ strains of $P$. aeruginosa isolated from hospitalised patients with lower respiratory tract infections in 2000-2001 [1]. Among isolates from France, the susceptibility rates for cefepime, ciprofloxacin and gentamicin were $<60 \%$, while for amikacin, ceftazidime, imipenem and piperacillin-tazobactam they were only $\sim 70 \%$. A similar pattern was seen for isolates from Italy, whereas those from Germany, Spain, Canada and the USA had somewhat higher susceptibility rates. Nevertheless, in these countries, susceptibility rates of $\geqslant 80 \%$ were seen only for amikacin and piperacillin-tazobactam [1]

Similar findings have been obtained in analyses of Acinetobacter species and MRSA. Against
Acinetobacter species isolated from ICU patients during 2000-2002, the susceptibility rates for third-generation cephalosporins, fluoroquinolones and gentamicin were $<50 \%$ for isolates from France, Italy and the USA, and $\sim 70 \%$ for isolates from Germany and Canada. Although carbapenems were the most active antibiotic class tested, susceptibility to meropenem and imipenem ranged $94-96 \%$ in Germany and Canada, and $75-78 \%$ in Italy [2]. Similarly, MRSA now accounts for a large proportion of $S$. aureus isolates from ICU patients. The prevalence of MRSA rose dramatically in the late 1990s in the remained at lower levels in other countries, such as Germany [3].

\section{INFLUENCE OF ANTIBIOTIC THERAPY ON THE EMERGENCE OF RESISTANCE}

The challenge presented by increasing resistance needs to be addressed by improving prevention policies and by making better use of antibiotic therapy, specifically by shortening treatment duration and lowering the amount of antibiotics administered to ICU patients. This may be the only way to contain the emergence and dissemination of multidrug-resistant pathogens. It is USA to $>50 \%$ among ICU isolates; however, it
CORRESPONDENCE

J. Chastre

Service de Réanimation Médicale Institut de Cardiologie Groupe Hospitalier Pitié-Salpêtrière 47 Boulevard de I'Hôpital 75651 Paris Cedex 13 France Fax: 33142163823 E-mail: jean.chastre@psl.aphp.fr

\section{STATEMENT OF INTEREST}

J. Chastre serves on the Nektar advisory board and has received speaker honoraria from Pfizer, Astra Zeneca, Wyeth, Pharm-Olam and Brahms.

C.E. Luyt has not declared a statement of interest. 
clear that the total amount of antibiotics used is directly related to the emergence of multidrug-resistant strains in the ICU. As shown in figure 1, the increase in fluoroquinolone use in the USA during the 1990s was directly and significantly related to the increasing frequency of ciprofloxacin resistance among ICU isolates of $P$. aeruginosa $(\mathrm{r}=0.96, \mathrm{p}<0.001)$ and Gram-negative bacilli $(r=0.89, p=0.007)$ [4]. Notably, ciprofloxacin resistance was associated with cross-resistance to other broad-spectrum antibiotics. Among ciprofloxacin-resistant $P$. aeruginosa isolates, resistance rates of $66,40,38$ and $26 \%$ were seen for gentamicin, ceftazidime, imipenem and amikacin, respectively [4]. Similarly, in a study conducted in 15 New York City hospitals (New York, NY, USA), the use of cephalosporins in combination with aztreonam was directly associated with the rates of ceftazidime- and carbapenem-resistant Acinetobacter baumannii at each hospital ( $\mathrm{p}=0.03$ and $\mathrm{p}=0.004$, respectively) [5].

Until recently, most experts recommended that the treatment of nosocomial infections should last $\geqslant 14$ days in most cases. This recommendation, however, remains largely empiric due to a lack of prospective controlled studies. Unfortunately, this traditional approach of prolonged antibiotic therapy may favour the emergence of multidrug-resistant strains, expose patients to unnecessary antibiotic toxicity and increase costs.

In a study of patients with ventilator-associated pneumonia (VAP) receiving appropriate antibiotic therapy for a median (range) duration of 13 (7-14) days, significant improvement in all clinical parameters was observed within the first 6 days of antibiotic therapy. Colonisation of endotrachial aspirates with $P$. aeruginosa, $S$. aureus and Enterobacteriaceae persisted despite in vitro susceptibility to the antibiotics used. Within a few days of therapy, emergence of new resistant strains developed, as shown in figure 2. By day 15, seven patients were newly colonised with $P$. aeruginosa, six with Enterobacteriaceae and four with $S$. aureus. Overall, six patients developed a recurrent episode of VAP, including four patients with $P$. aeruginosa. In three of the cases caused by $P$. aeruginosa and in the lone case due to Escherichia coli, the isolate was resistant to the antibiotic used initially [6]. These findings illustrate that prolonging the duration of antibiotic therapy in VAP patients favours the emergence of multidrug-resistant strains in the proximal airways. This is likely to occur without any apparent benefit on the clinical course of disease.

\section{OPTIMAL DURATION OF ANTIBIOTIC THERAPY IN VAP. SHORT-TERM THERAPY}

The optimal duration of antibiotic therapy in VAP was explored in a large, prospective, randomised, double-blind study conducted at 51 ICUs in France [7]. Adults with a clinical diagnosis of VAP documented by positive quantitative cultures of distal bronchoscopic specimens were eligible if they had started appropriate empirical antibiotic therapy within $24 \mathrm{~h}$ after bronchoscopy. Antibiotic therapy was considered appropriate if it provided coverage against the pathogen(s) responsible for the infection based on in vitro susceptibility patterns. Of the patients screened, 401 were randomly assigned at day 3 to continue antibiotic therapy for a total of $8(n=197)$ or 15 days $(n=204)$. The investigators and the patients remained blind to antibiotic therapy until day 8 , at which time the treatment assignment was communicated to the investigators.

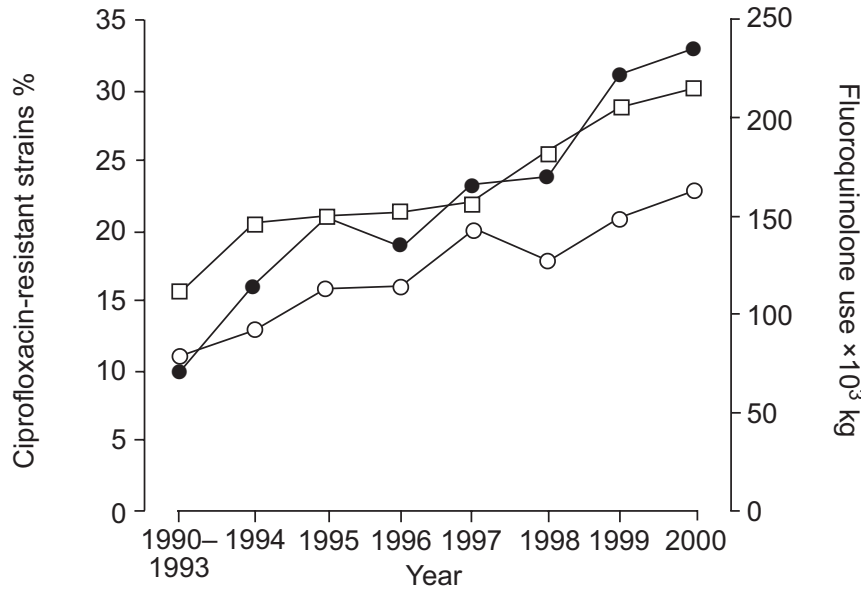

FIGURE 1. Relationship between fluoroquinolone use $(\square)$ and resistance rates in Pseudomonas aeruginosa ( $)$ and Gram-negative bacilli $(\bigcirc)$ in the USA during the 1990s. Reprinted from [4] with permission from the publisher.

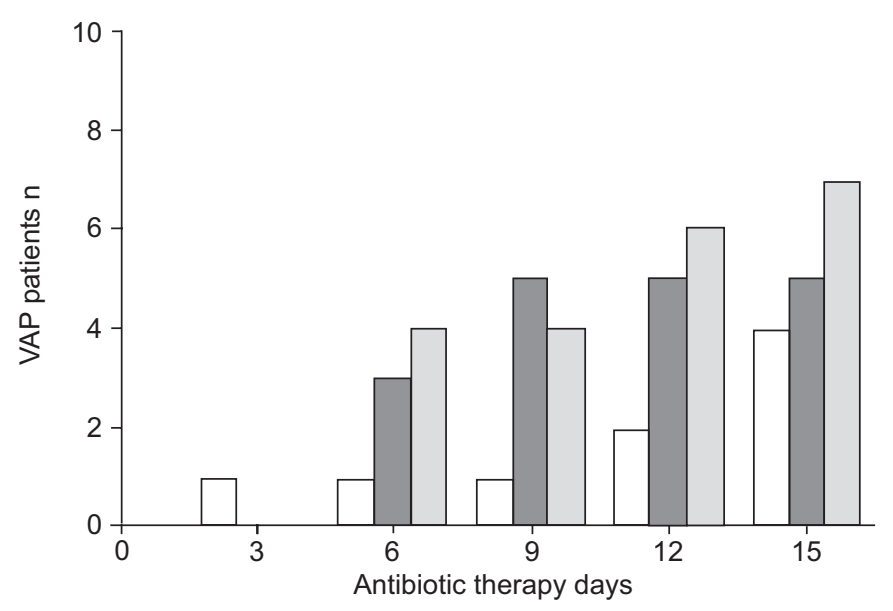

FIGURE 2. Number of ventilator-associated pneumonia (VAP) patients with newly isolated micro-organisms from endotracheal aspirates after initiation of antibiotic treatment. $\square$ : Staphylococcus aureus; $\square$ : Enterobacteriaceae; $\square$ : Pseudomonas aeruginosa. Reprinted from [6] with permission from the publisher.

The demographic and clinical characteristics of the groups assigned to receive antibiotic therapy for 8 or 15 days were generally similar, and the pathogens isolated in the two groups, as well as the initial antibiotic used, did not differ significantly [7]. The majority of patients in both groups initially received a regimen consisting of a $\beta$-lactam antibiotic in combination with either an aminoglycoside or a fluoroquinolone $(91 \%$ of patients in the 8 -day group and $92 \%$ of patients in the 15-day group). Figure 3 illustrates that prolonging the duration of antibiotic therapy to 15 versus 8 days did not significantly influence the primary patient outcome: the probability of survival for 60 days after VAP onset [7]. The 28-day mortality rates following VAP onset were 18.8 and $17.2 \%$ in the 8 - and 15 -day groups, respectively, and the rates of microbiologically documented recurrent pulmonary infection were no different, 28.9 versus $26.0 \%$, respectively $(2.9 \%$ difference; $90 \%$ confidence interval (CI) -3.2-9.1). Therefore, patients treated with antibiotics for 8 days do not experience 
greater mortality or greater risk of recurrent infection than those treated for 15 days.

The clinical responses did not differ between the 8- and 15-day groups, with equivalent improvements in fever, leukocyte count, arterial oxygen pressure/inspiratory oxygen fraction $\left(\mathrm{Pa}, \mathrm{O}_{2} / \mathrm{FI}_{1} \mathrm{O}_{2}\right)$ ratio, organ dysfunction and radiological scores. Nevertheless, the price of prolonging antibiotic therapy to 15 days was seen among patients who developed recurrent pulmonary infections. Multidrug-resistant pathogens emerged significantly more frequently: there were $62.3 \%$ versus $42.1 \%$ recurrent infections in patients who received 15 versus 8 days of antibiotic therapy $(\mathrm{p}=0.038)$ [7].

The duration of antibiotic therapy decreased significantly following the implementation of a clinical guideline for the treatment of VAP in a university-affiliated medical ICU in the USA [8]. A total of 102 VAP patients were followed prospectively, i.e. 50 consecutive patients before implementation of the guideline and 52 consecutive patients afterwards. Patients in both groups had similar disease severity and clinical pulmonary infection scores. Following guideline implementation, patients were significantly more likely to receive appropriate empirical antibiotic therapy (94.2 versus $48.0 \%$, p $<0.001$ ), which was administered for a significantly shorter duration (8.6 versus 14.8 days, $p<0.001)$. The severity of illness and clinical pulmonary infection scores were similar during the two treatment periods. However, VAP recurrent episodes declined significantly following guideline implementation $(7.7 \%$ after implementation versus $24.0 \%$ before, $\mathrm{p}=0.03$ ) [8].

\section{OPTIMAL DURATION OF ANTIBIOTIC THERAPY IN VAP: LONG-TERM THERAPY}

Several types of patients with VAP may require antibiotic therapy for $>8$ days. These include: immunocompromised patients; patients who have received inappropriate empirical antibiotic therapy; patients with infections caused by difficult-totreat pathogens; and patients with a high probability of relapse. Although patients who had received inappropriate empirical therapy were excluded from the large prospective French study described previously [7], patients with difficult-to-treat

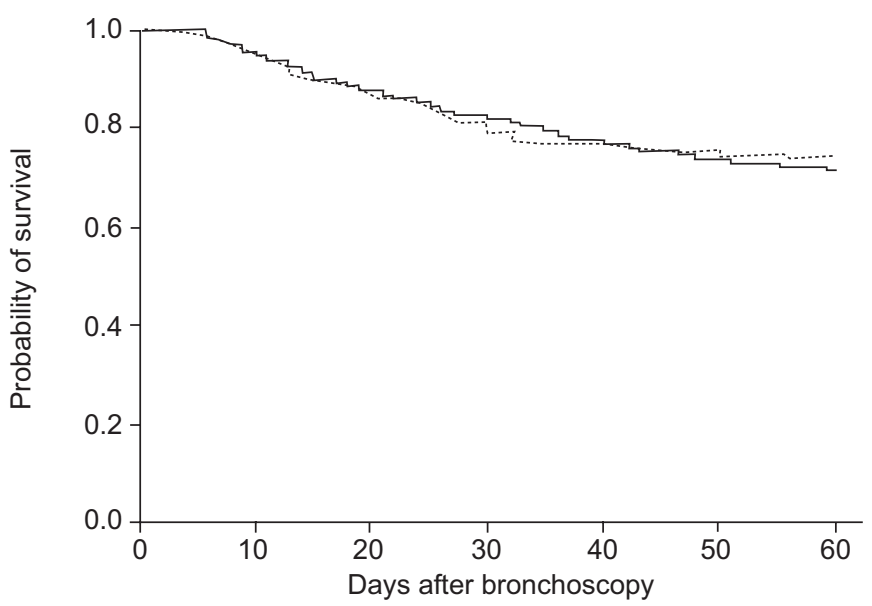

FIGURE 3. Cumulative survival estimates according to duration of antimicrobial treatment: 8-day $(\cdots \cdots)$ versus 15-day $(-)$ regimens. Reprinted from [7] with permission from the publisher. infections were included. Overall, $32.9 \%$ of patients in the 8-day group and $30.9 \%$ of patients in the 15-day group had VAP caused by nonfermentative Gram-negative bacilli, primarily $P$. aeruginosa. The 28-day mortality rate did not differ significantly between the two groups with nonfermentative Gram-negative bacilli: $23.4 \%$ in the 8 -day group and $30.2 \%$ in the 15 -day group (-6.8\% difference; $90 \%$ CI: $-17.5-4.1$; fig. 4). The clinical response did also not differ, but the rate of recurrent pulmonary infection was higher in the 8-day group than in the 15-day group: 40.6 versus $25.4 \%$, respectively (15.2\% difference; $90 \%$ CI: $3.9-26.6$; $\mathrm{p}=0.09$ ) [7]. Therefore, in patients with VAP caused by nonfermentative Gram-negative bacilli, it may be necessary to use longer antibiotic therapy.

Stepwise logistic regression analysis found four factors independently associated with an unfavourable outcome in VAP patients of either death or recurrent pulmonary infection [9]. These were: 1) baseline Simplified Acute Physiological Score II at admission ( $\mathrm{p}=0.007) ; 2$ ) day 7 radiological score $(p=0.0004) ; 3)$ day 7 Sepsis-related Organ Dysfunction Assessment Score $(p=0.04)$; and 4$)$ the need to prolong mechanical ventilation beyond day $7(p=0.03)$. Pending the results of prospective studies designed to evaluate the optimal duration of antibiotic therapy and providing empirical antibiotic therapy is appropriate, it can be concluded that it is safe to administer antibiotics for 8 days to patients with VAP caused by nonfermentative Gram-negative bacilli. Extreme vigilance is imperative after antibiotic therapy is stopped. This clinical course is favourable, particularly when it is possible to wean the patient from mechanical ventilation before the end of antibiotic therapy [9].

\section{INDIVIDUALISING THE DURATION OF ANTIBIOTIC THERAPY BASED ON PATIENT STATUS AND RISK FACTORS}

It may be possible to customise the duration of antibiotic therapy according to the patient's clinical status and risk factors for an adverse outcome. The feasibility of basing antibiotic duration on the patient's clinical status was

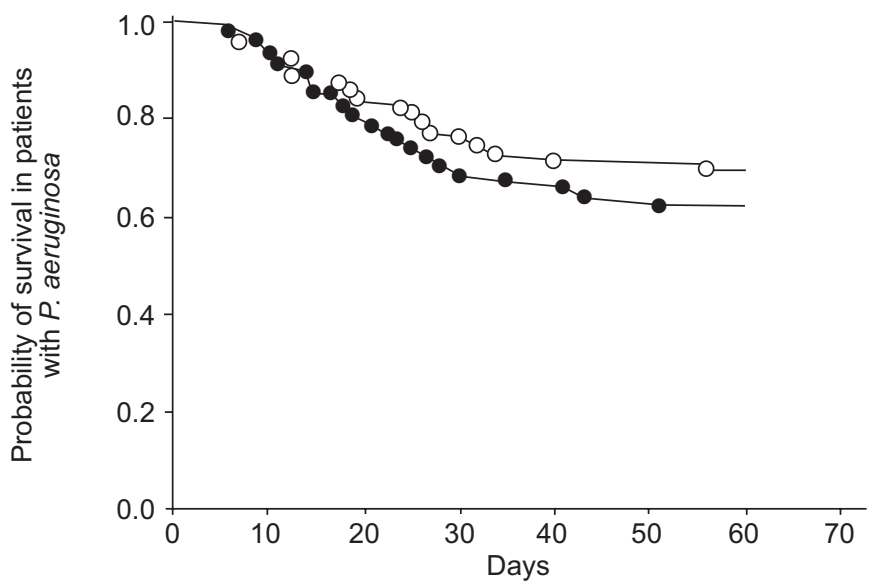

FIGURE 4. Cumulative survival estimates for the subgroup of 127 patients with ventilator-associated pneumonia caused by nonfermentative Gram-negative bacilli according to the duration of antimicrobial treatment: 8-day ( $\bigcirc)$ versus 15-day ( regimens. $P$. aeruginosa: Pseudomonas aeruginosa. 
evaluated in a prospective, randomised, controlled trial of 302 patients with VAP in whom the duration of antibiotic therapy was randomly determined by either an antibiotic discontinuation policy or by the treating physician [10]. In the policy group, recommendations were made to stop antibiotics if the infiltrate was identified as noninfectious and if the signs and symptoms suggesting an active infection had resolved (body temperature $<38.3^{\circ} \mathrm{C}$, leukocyte count $<10,000$ cells $\mu \mathrm{L}^{-1}$ or $\geqslant 25 \%$ below peak values, absence of purulent sputum, improvement or lack of progression on the chest radiography and $\mathrm{Pa}, \mathrm{O}_{2} / \mathrm{FI}_{1} \mathrm{O}_{2}>250$ ). Adequate empirical antibiotic therapy was administered to $93 \%$ of patients in both groups [10].

In figure 5 , the duration of antibiotic therapy was significantly shorter in the discontinuation policy group (6 versus 8 days, $p=0.001)$, and the duration of therapy correlated significantly with clinical pulmonary infection scores $(r=0.42, p<0.001)$ [10]. Antibiotic therapy was longer in patients with clinical pulmonary infection scores of 10 and shorter in patients with scores $\leqslant 6$. Both groups had similar clinical outcomes, including hospital mortality (37.1 versus $32.0 \%, \mathrm{p}=0.36$ ), length of hospital stay (15.4 versus 15.7 days, $\mathrm{p}=0.87$ ), length of ICU stay ( 7.0 versus 6.8 days, $\mathrm{p}=0.80)$, duration of mechanical ventilation (5.7 versus 5.4 days, $\mathrm{p}=0.65$ ) and proportion experiencing re-infection (32.9 versus $37.3 \%, p=0.43$ ). Therefore, it is possible to shorten the duration of antibiotic therapy in many patients with VAP without compromising the clinical outcome.

The value of specific risk factors in customising the duration of antibiotic therapy remains to be demonstrated. Several clinical and biological factors have been shown to evolve differently among survivors and nonsurvivors of VAP. For example, starting on day 3 after VAP diagnosis, serial measurements of the modified clinical pulmonary infection score were able to distinguish between survivors and nonsurvivors in a cohort of 63 patients managed in the ICUs of six hospitals in Buenos Aires, Argentina [11]. Of the individual components of the clinical pulmonary infection score, only the improvement observed in $\mathrm{Pa}_{1} \mathrm{O}_{2} / \mathrm{FI}_{1} \mathrm{O}_{2}$ significantly distinguished survivors from nonsurvivors.

In a separate study of 63 consecutive patients managed in a medical ICU, elevated serum procalcitonin levels on days 1-7 were independently associated with an unfavourable outcome (death, VAP recurrence or extrapulmonary infection requiring antibiotics before day 28). Multivariate analysis showed procalcitonin levels on days 1, 3 and 7 as strong predictors of an unfavourable outcome [12].

\section{CONCLUSION}

Antibiotics in the intensive care unit are a dual-edged sword. Clearly, they are important for promoting the clinical cure of ventilator-associated pneumonia and may confer additional benefit in selected patients by reducing the incidence of early relapse. However, prolonging the duration of antibiotic therapy confers a significant risk for the emergence of resistant micro-organisms and more severe late-onset infection. Therefore, there is a need in clinical practice to minimise the duration of antibiotic therapy. This important point is advocated in the official statement of the American Thoracic Society and the Infectious Diseases Society of America for managing patients with ventilator- or healthcare-associated

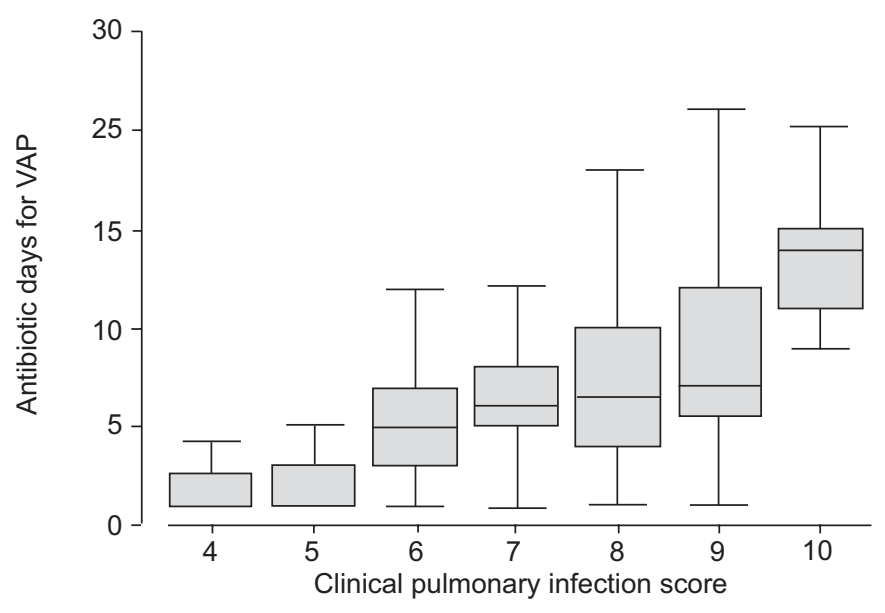

FIGURE 5. Duration of antibiotic therapy according to clinical pulmonary infection score in a randomised trial of an antibiotic discontinuation policy for clinically suspected ventilator-associated pneumonia (VAP). $r=0.419 ; p<0.001$. Reprinted from [10] with permission from the publisher.

pneumonia [13]. For patients treated initially with an appropriate antibiotic regimen, "efforts should be made to shorten the duration of therapy from the traditional 14-21 days to periods as short as 7 days, providing that the aetiological pathogen is not Pseudomonas aeruginosa, and that the patient has a good clinical response with resolution of clinical features of infection." This recommendation is based on level I evidence, meaning that it is supported by well-conducted, randomised controlled trials.

\section{REFERENCES}

1 Wenzel RP, Sahm DF, Thornsberry C, Draghi DC, Jones ME, Karlowsky JA. In vitro susceptibilities of gramnegative bacteria isolated from hospitalized patients in four European countries, Canada, and the United States in 2000-2001 to expanded-spectrum cephalosporins and comparator antimicrobials: implications for therapy. Antimicrob Agents Chemother 2003; 47: 3089-3098.

2 Jones ME, Draghi DC, Thornsberry C, Karlowsky JA, Sahm DF, Wenzel RP. Emerging resistance among bacterial pathogens in the intensive care unit-a European and North American Surveillance study (2000-2002). Ann Clin Microbiol Antimicrob 2004; 3: 14.

3 Harbarth S, Albrich W, Goldmann DA, Huebner J. Control of multiply resistant cocci: do international comparisons help? Lancet Infect Dis 2001; 1: 251-261.

4 Neuhauser MM, Weinstein RA, Rydman R, Danziger LH, Karam G, Quinn JP. Antibiotic resistance among gramnegative bacilli in US intensive care units: implications for fluoroquinolone use. JAMA 2003; 289: 885-888.

5 Landman D, Quale JM, Mayorga D, et al. Citywide clonal outbreak of multiresistant Acinetobacter baumannii and Pseudomonas aeruginosa in Brooklyn, NY: the preantibiotic era has returned. Arch Intern Med 2002; 162: 1515-1520.

6 Dennesen PJ, van der Ven AJ, Kessels AG, Ramsay G, Bonten MJ. Resolution of infectious parameters after antimicrobial therapy in patients with ventilator-associated pneumonia. Am J Respir Crit Care Med 2001; 163: 1371-1375. 
7 Chastre J, Wolff M, Fagon JY, et al. Comparison of 8 versus 15 days of antibiotic therapy for ventilator-associated pneumonia in adults: a randomized trial. JAMA 2003; 290: 2588-2598.

8 Ibrahim EH, Ward S, Sherman G, Schaiff R, Fraser VJ, Kollef $\mathrm{MH}$. Experience with a clinical guideline for the treatment of ventilator-associated pneumonia. Crit Care Med 2001; 29: 1109-1115.

9 Combes A, Luyt CE, Fagon JY, Wolff M, Trouillet JL, Chastre J. Early predictors for infection recurrence and death in patients with ventilator-associated pneumonia. Crit Care Med 2007; 35: 146-154.

10 Micek ST, Ward S, Fraser VJ, Kollef MH. A randomized controlled trial of an antibiotic discontinuation policy for clinically suspected ventilator-associated pneumonia. Chest 2004; 125: 1791-1799.

11 Luna CM, Blanzaco D, Niederman MS, et al. Resolution of ventilator-associated pneumonia: prospective evaluation of the clinical pulmonary infection score as an early clinical predictor of outcome. Crit Care Med 2003; 31: 676-682.

12 Luyt CE, Guerin V, Combes A, et al. Procalcitonin kinetics as a prognostic marker of ventilator-associated pneumonia. Am J Respir Crit Care Med 2005; 171: 48-53.

13 American Thoracic Society; Infectious Diseases Society of America. Guidelines for the management of adults with hospital-acquired, ventilator-associated, and healthcareassociated pneumonia. Am J Respir Crit Care Med 2005; 171: 388-416. 